\title{
Oculo-Respiratory Syndrome
}

National Cancer Institute

\section{Source}

National Cancer Institute. Oculo-Respiratory Syndrome. NCI Thesaurus. Code C116333.

A transient condition that can occur following influenza vaccination that is characterized

by bilateral conjunctivitis, facial edema, and upper respiratory symptoms. 